\title{
Visual discrimination thresholds for time to arrival
}

\author{
Klaus Landwehr • Robin Baurès • Daniel Oberfeld • \\ Heiko Hecht
}

Published online: 22 June 2013

(C) Psychonomic Society, Inc. 2013

\begin{abstract}
In a seminal article, Todd (Journal of Experimental Psychology: Human Perception and Performance 7:795$810,1981)$ reported a difference threshold of about $50 \mathrm{~ms}$ to discriminate the times of arrival of two differently sized objects that simultaneously approached head-on at constant but different velocities. Subsequent investigators, however, have often found much higher thresholds. We did one complete replication of Todd's experiment, and then modified his stimuli and experimental regime, which we hypothesized may have been responsible for some of the discrepancies reported in the literature. Unlike Todd and most other researchers, we exclusively used untrained observers. Several of our participants performed almost as well as the trained observers used by Todd and others, but the performance of most of our participants fell short of this standard. Furthermore, thresholds were affected by the experimental regimes, with large differences between objects' sizes and speeds compromising performance. Analyses of the response patterns revealed that the responses were driven mainly by the objects' relative apparent sizes.
\end{abstract}

Keywords Visual perception · Optic flow · Time to collision $\cdot$ Difference thresholds

Time to arrival $\left(t_{\mathrm{A}}\right)$ is the time remaining before an object or an observer, moving toward a goal, will actually arrive there (Schiff \& Oldak, 1990). Here, we shall be concerned with the ability of human observers to visually discriminate between different $t_{\mathrm{A}} \mathrm{s}$. Three decades ago, Todd (1981) reported that the

K. Landwehr $\cdot$ R. Baurès $\cdot$ D. Oberfeld $\cdot H$. Hecht

Psychologisches Institut, Universität Mainz, Mainz, Germany

K. Landwehr $(\bowtie)$

Psychologisches Institut, Universität Mainz, 55099 Mainz, Germany

e-mail: landweh@uni-mainz.de discrimination threshold for the times of arrival of two objects, which simultaneously approached head-on toward a stationary observer, amounted to a mere $50 \mathrm{~ms}{ }^{1}{ }^{1}$ Most subsequent work, however, has obtained values seven to ten times higher than this (Table 1). We think that it is important to identify the causes of these apparent discrepancies, because otherwise our estimates of human proficiency with regard to $t_{\mathrm{A}}$ encounters might possibly be too optimistic. In this article, we shall focus on three ingredients of $t_{\mathrm{A}}$ experiments that might explain the observed discrepancies: (a) the use of different stimuli, (b) the use of different experimental regimes, and (c) the use of differently qualified observers.

\section{Stimuli}

Most research on visual discrimination of arrival times makes reference to the geometrical-optical variable $\tau$, defined as the ratio of a given visual angle and its instantaneous first temporal derivative (Lee, 1974, 1976). For comparatively small visual angles, head-centered straight trajectories, and constant velocity, $\tau$ specifies $t_{\mathrm{A}}$. As was explained by Lee and Young (1985), there are three possibilities to define visual angles that may be entered into the $\tau$ formula: Reference is to two points on a plane surface, or to an object's outer contour, or to the socalled focus of expansion of an optic flow field considered in its entirety (cf. Gibson, 1950). The three types of $\tau$ that ensue were dubbed local $\tau$ type $1\left(\tau_{\mathrm{L}}^{(1)}\right)$, local $\tau$ type $2\left(\tau_{\mathrm{L}}^{(2)}\right)$, and global $\tau\left(\tau_{\mathrm{G}}\right)$, respectively, by Tresilian (1991). For head-

\footnotetext{
${ }^{1}$ As has also been noted by others (e.g., Bootsma \& Craig, 2002, p. 918, note 6), in Todd's (1981) experiment, approach was not head-on in the sense of the two objects' midpoints traveling on converging trajectories toward the observer (cf. Kebeck \& Landwehr, 1992, for such a scenario); rather, the two objects were set side by side, so that the two abutting lateral edges traveled on a collision course along the observer's cyclopean line of gaze.
} 
Table 1 Authors, parameters, and results of previous $t_{\mathrm{A}}$ discrimination threshold studies

\begin{tabular}{|c|c|c|c|c|c|}
\hline Authors & Paradigm & Stimuli & Observers & Thresholds (ms) & Weber Fractions \\
\hline Todd, 1981 & $2 \mathrm{AFC}$ & Dotted-outline squares & Trained & 50 & 0.016 \\
\hline Simpson, 1988 & 2AFC-Stc & Crosses & Trained/naïve & $195 \sim 443_{\mathrm{r}}$ & $0.15 \sim 0.34$ \\
\hline Bootsma \& Oudejans, 1993 & $2 \mathrm{AFC}$ & Outline squares & Trained & $50_{\mathrm{f}}$ & 0.02 \\
\hline Kaiser \& Mowafy, 1993 & $2 \mathrm{AFC}$ & Dots & Informed & $500_{\mathrm{g}}$ & 0.125 \\
\hline Regan \& Hamstra, 1993 & 1I-2AFC & Filled squares & Trained & $32 \sim 85$ & $0.049 \sim 0.129$ \\
\hline Bootsma \& Craig, 2002 & $2 \mathrm{AFC}$ & Spheres/dots & Naïve & $250_{\mathrm{g}} \sim 500$ & $0.083 \sim 0.125$ \\
\hline Kim \& Grocki, 2006 & $2 \mathrm{AFC}$ & Hexahedrons & Naïve & 500 & 0.25 \\
\hline
\end{tabular}

$2 \mathrm{AFC}=$ Two-alternative forced choice. Stc $=$ Staircase. $1 \mathrm{I}=$ One interval. Unmarked thresholds refer to approach events, those indexed with an " $\mathrm{f}$ " to frontoparallel motion, those indexed with an " $\mathrm{r}$ " to recession events, and those indexed with a "g" to $\tau_{\mathrm{G}}$-defined events. Weber fractions were computed by dividing the thresholds by mean $t_{\mathrm{A}}$ - or by mean viewing time $\left(t_{\mathrm{view}}\right)$, if $t_{\mathrm{A}}>>t_{\text {view. }}$. The threshold reported by Todd (1981) was read off his Fig. 3, the one reported by Kaiser and Mowafy (1993) from their Fig. 4, the ones reported by Bootsma and Craig (2002) from their Figs. 4-6, and the one reported by Kim and Grocki (2006) from their Fig. 3, all in accordance with a 75 \%-correct criterion. The thresholds reported by Regan and Hamstra (1993) were independent from $t_{\mathrm{A}}$.

centered, straight trajectories, the optic flow field's focus of expansion coincides with the object's or the observer's direction of travel and gaze (Warren, 1998), and global $\tau$, at this point, is undefined (or zero). It is only with off-center passages that $\tau_{\mathrm{G}}$ comes into play (cf. Kaiser \& Mowafy, 1993, who isolated this variable by using dot-like objects). Geometrically, $\tau_{\mathrm{L}}^{(1)}$ cannot be deconfounded from $\tau_{\mathrm{L}}{ }^{(2)}$ (Beverley \& Regan, 1980, p. 158: Fig. 5), but visual systems may respond differently to continuous contours than to discrete dots. If so, $\tau_{\mathrm{L}}{ }^{(1)}$ can be isolated by means of two dots, and $\tau_{\mathrm{L}}{ }^{(2)}$, by means of a closed contour - the circle, in particular, because it does not exhibit singular points.

Most of the objects that have been used in $t_{\mathrm{A}}$ research (e.g., filled or outline squares, hexahedrons; Table 1) did not perfectly deconfound $\tau_{\mathrm{L}}{ }^{(1)}$ and $\tau_{\mathrm{L}}{ }^{(2)}$, in the sense just explained, but emphasized $\tau_{\mathrm{L}}^{(2)}$; only Simpson's (1988) crosses nearly isolated $\tau_{\mathrm{L}}{ }^{(1)}$-although the four corners of a square or the maximally visible six or seven corners of an hexahedron pairwise provide obvious $\tau_{\mathrm{L}}{ }^{(1)}$ information, and, conversely, the four outermost points of a cross constitute a virtual square, hence, $\tau_{\mathrm{L}}{ }^{(2)}$ information. Todd's (1981) dotted-outline squares (cf. the first paragraph of the Prestudies section for a precise characterization) occupy an intermediate territory: For one thing, a dense sequence of dots strongly suggests the presence of a line (Westheimer \& Li, 1996); for another, four virtual, orthogonal lines strongly suggest a square (Kanizsa, 1979). ${ }^{2}$ Todd's (1981) stimuli thus provide both types of local $\tau$ information simultaneously. With regard to stimuli, we reasoned that if the superior performance of Todd's observers had been due to the available stimulus information, then random arrays of dots with the same number of flow vectors as were

\footnotetext{
${ }^{2}$ Our reasoning may be reminiscent of phenomenological Gestalt theory here, but we note that there is neurophysiological evidence that our visual system under specific conditions responds according to "laws of grouping," or even principles of "amodal completion" (Roelfsema, 2006; Sasaki, 2007).
}

present in Todd's squares would constitute an appropriate control stimulus: Such "dot clouds," as we shall call them, would compromise $\tau_{\mathrm{L}}{ }^{(2)}$, emphasize $\tau_{\mathrm{L}}{ }^{(1)}$, but retain dense optic flow (Fig. 1). Thence, on the assumption that $\tau_{\mathrm{L}}{ }^{(2)}$ information can be dispensed with in favor of $\tau_{\mathrm{L}}{ }^{(1)}$, the cloud stimuli should support $t_{\mathrm{A}}$ performance as well as Todd's original stimuli did.

It is important to note that our cloud stimuli differ from those that have typically been used to study effects of surface texture on $t_{\mathrm{A}}$ judgments (cf. Landwehr, 2004, for a review; Hosking \& Crassini, 2011; Jacobs \& Díaz, 2010; LópezMoliner, Brenner, \& Smeets, 2007; Oberfeld, Hecht, \& Landwehr, 2011, for more recent research). In the respective experiments, "texture" has usually been materialized by checkerboard tilings. Although, geometrically speaking, in any such display an infinity of pairs of points define plane visual angles, dedicated response mechanisms are more likely to be activated by points that stand out optically (Beverley \& Regan, 1979, 1980). The observed effects of texture have most often been weak, even when dot patterns have been used (DeLucia, Kaiser, Bush, Meyer, \& Sweet, 2003). Harris and Giachritsis (2000; Giachritsis \& Harris, 2005), who also used random-dot kinematograms, found that, with regard to $t_{\mathrm{A}}$ judgments, global image expansion dominated over local, dot-related size changes. However, in these experiments, levels of information were pitted against one another, and in terms of $\tau$ variables, the comparison was between $\tau_{\mathrm{G}}$ and $\tau_{\mathrm{L}}{ }^{(2)}$, or between $\tau_{\mathrm{G}}$ and a mix of $\tau_{\mathrm{L}}{ }^{(1)}$ and $\tau_{\mathrm{L}}{ }^{(2)}$. We therefore think that a comparison of stimuli that emphasized either $\tau_{\mathrm{L}}^{(1)}$ and $\tau_{\mathrm{L}}^{(2)}$, or $\tau_{\mathrm{L}}{ }^{(1)}$ alone (with $\tau_{\mathrm{G}}$ constant), would still be informative.

\section{Experimental regimes}

Inspection of Table 1 does not reveal any obvious differences between the experimental paradigms that have been used by 


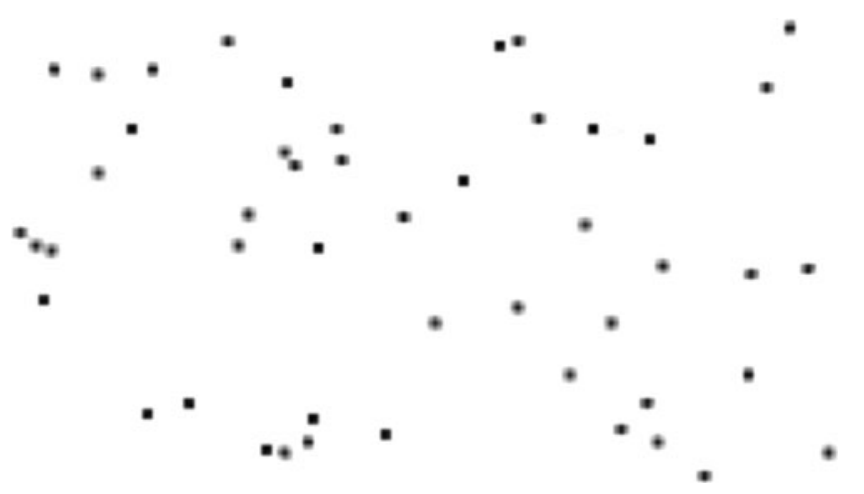

Fig. 1 Screenshot of our dot-cloud stimulus

individual researchers or research groups: All took advantage of a variant of the two-alternative forced choice paradigm (2AFC; Jones, 1971). Regan and Hamstra (1993), however, used a one-interval stimulus presentation procedure and an implicit standard (the arithmetic mean of a series of $t_{\mathrm{A}}$ values, to be discovered "online" by observers themselves; cf. McKee, 1981), ${ }^{3}$ and Simpson (1988) combined the basic paradigm with a maximum-likelihood staircase procedure (Pentland, 1980). Such modifications of the 2AFC paradigm may affect performance (see Macmillan \& Creelman, 2005, pp. 175-176), although, with regard to the use of an implicit standard, Morgan, Watamaniuk, and McKee (2000) have shown an explicit standard to be superfluous (see also Norman et al., 2008).

A systematic comparison of possible variants of $2 \mathrm{AFC}$ was beyond our present endeavor. Instead, we concentrated on the stimulus variants described in the preceding section, and on observer variables (to be discussed subsequently), and modeled our experimental setup strictly after Todd's (1981). However, a number of observations that accrued during prestudies (cf. the Prestudies section of this article) prompted us to consider various modifications of Todd's original experimental regime. Table 2 provides a contrasting juxtaposition of Todd's regime and the one that we used, for purposes of comparison in our main experiment. The most important differences between the regimes concern the presence versus absence of a fixed standard, partial versus complete randomization of the sequence of trials, and different ranges of object sizes and velocities. As we shall see from the results of our main experiment, some of these modifications can be held responsible for the observed differences in participants' performance.

\footnotetext{
${ }^{3}$ In Macmillan and Creelman's (2005, pp. 113-120) systematization, this procedure is regarded as a two-response classification task that presupposes the perceptual one-dimensionality of the stimulus. Note that these authors also assigned Fechner's (1860) method of constant stimuli- the method used by most of the authors listed in Table 1 (plus ourselves) - to this category.
}

\section{Observers}

With regard to the observers' status, most of the authors cited in Table 1 had recruited trained observers, including themselves (e.g., Regan \& Hamstra, 1993; Todd, 1981). This raises concerns about population validity. In fact, Simpson (1988) reported differences between his own data and those of a group of naïve observers, as well as deviating results for another, individual untrained observer (related to symmetric vs. asymmetric sensitivity to approach vs. recession). Not surprisingly, expert observers generally outperform the others. The use of trained observers is meaningful if one's aim is "testing the limits," and indeed there are remarkable achievements of timely responses in a variety of sports (cf., e.g., Bootsma \& van Wieringen, 1990; Regan, 2012). However, the use of naïve observers is called for if one is interested in the average performance of lay persons. We invited undergraduate students, and only accepted those who had not participated in a $t_{\mathrm{A}}$ experiment before.

\section{Prestudies}

All of the studies to be described in this article were done as computer simulations. We did one complete replication of Todd's (1981) Experiment 1. As in the original, two squares, set side by side with the midpoints of their abutting edges centered on the observer's cyclopean line of gaze, moved head-on toward him or her. The simulated object sizes $(R)$ were 7.6 and $38.1 \mathrm{~cm}$, the velocities $(v)$ were $6.1,9.1,12.2$, $15.2,18.3,21.3,24.4$, and $27.4 \mathrm{~m} \cdot \mathrm{s}^{-1}$, the arrival time $\left(t_{\mathrm{A}}\right)$ of the standard was $3 \mathrm{~s}$, and the $t_{\mathrm{A}} \mathrm{s}$ of the test were $3.3,3.2,3.15$, $3.1,3.05,3.02$, and $3.01 \mathrm{~s}$, respectively. A total of 512 unique trials could be generated by randomly drawing pairs of $R$ and $v$, and by interchanging the left-right positions of the standard and test. The viewing distance was $76.2 \mathrm{~cm}$ for a screen size of $21.6 \times 16.5 \mathrm{~cm}$. Objects were optically specified by $2 \times 24$ dots, spaced evenly along the objects' outer contours. Dots did not magnify during approach, and squares did not deform, thus corresponding to parallel projection. ${ }^{4}$ The stimuli were turned off shortly after the edges of one object had hit the edges of the screen. One lay observer, who had not taken part in a similar experiment before, participated. As in Todd's original experiment, the participant was asked to respond "as quickly as possible without sacrificing accuracy" (p. 799), and she was informed that responses would be recorded only as long as both objects were completely visible — and that invalid

\footnotetext{
${ }^{4}$ Todd (1981) had generated his stimuli not trigonometrically, but according to a hyperbolic approximation (object size divided by distance), and, for the sake of comparability, so did we. Although this compromises quantitative analyses of $\tau$-type information in terms of visual angles, it leaves the qualitative differences between the types of $\tau$, as materialized in the stimuli, unaltered.
} 
Table 2 A comparison of Todd's (1981) original experimental regime and our new regime

\begin{tabular}{lll}
\hline & Todd's original regime & Our new regime \\
\hline Object sizes $(\mathrm{m})$ & 0.076 and/or 0.381 & $0.15 \sim 0.25$ \\
Ranges of visual angles at start $(\mathrm{deg})$ & $0.0017 \sim 3693$ & $0.0049 \sim 4.8777$ \\
Velocities $\left(\mathrm{m} \cdot \mathrm{s}^{-1}\right)$ & $6.1 \sim 27.4$ (in steps of $3 \sim 3.1)$ & $1.19 \sim 1.31(\equiv$ Pedestrian) \\
& & $3.56 \sim 3.94(\equiv$ Bicycle) \\
& & $10.69 \sim 11.81$ (三 Car in town) \\
& $17.9 \sim 273.7$ & $5.4 \sim 174.7$ \\
Ranges of angular velocities shortly before end $\left(\mathrm{deg} \cdot \mathrm{s}^{-1}\right)$ & 3 & $1.5 \sim 2.5$ \\
Standard $t_{\mathrm{A}}(\mathrm{s})$ & $0.01 \sim 0.3$ & $0.05 \sim 0.15$ (in steps of 0.025$)$ \\
$\Delta t_{\mathrm{A}} \mathrm{s}(\mathrm{s})$ & $($ in steps of $0.01 \sim 0.1)$ & 250 \\
Number of trials & 1,050 & Random highway) \\
Order of trials & According to $\Delta t_{\mathrm{A}}$ &
\end{tabular}

$\Delta t_{\mathrm{A}}=$ Difference between $t_{\mathrm{A}} \mathrm{S}$ of the standard and test. Except for $t_{\mathrm{A}}$ and $\Delta t_{\mathrm{A}}$, pairwise sampling from the ranges was random throughout

trials would be repeated at the end of a session (this only happened on $1.6 \%$ of trials). Answers were given by pressing a right-hand response key if the object on the right side was judged to arrive first, and by pressing a left-hand response key in the alternative case. Feedback was not provided because Todd's observers seem to have ignored it most of the time. The procedure was spatial $2 \mathrm{AFC}$ for a total of 1,050 trials that were ordered into 21 blocks with three repetitions of each arrivaltime difference $\left(\Delta t_{\mathrm{A}}\right)$ and $\Delta t_{\mathrm{A}}$ decreasing. Sessions were distributed over three consecutive days.

Contrary to the results with Todd's (1981) trained observers, our participant did not reach ceiling at $\Delta t_{\mathrm{A}}=$ $300 \mathrm{~ms}$, nor did her performance drop below chance at $\Delta t_{\mathrm{A}}$ $<50 \mathrm{~ms}$. Rather, it improved during the final blocks, and was still $57 \%$ correct at $\Delta t_{\mathrm{A}}=10 \mathrm{~ms}$. More importantly, on average, she scored $70 \%$ correct on trials with no size difference between the objects (i.e., $\Delta R=0$ ), $75 \%$ correct on trials with identical velocities $(\Delta v=0)$, and $90 \%$ correct on trials with both $\Delta R=0$ and $\Delta v=0$. These observations suggest that differently parameterized trials were handled differently by our participant (or posed different tasks in the first place), and that the whole experiment provided ample opportunity for perceptual learning.

Although we had used Todd's (1981) original instruction, our participant soon found out that responding as late as possible was advantageous: Her distribution of response times was extremely skewed, with a singular mode at $2.578 \mathrm{~s}$. As our participant revealed afterward, her strategy had been occasioned by trials in which she saw the apparently smaller, but faster, object overtaking the apparently larger one in the very last moment before stimulus wipeout. Also, she claimed to have benefited from becoming familiar with the average trial duration. Finally, fitting a psychometric function for trials with both $\Delta R \neq 0$ and $\Delta v \neq 0$ (cf. Wichmann $\&$ Hill, 2001) yielded a just noticeable difference (JND) of
$240 \mathrm{~ms}$ (Weber fraction: 0.08). After deselection of the first and final $\Delta t_{\mathrm{A}}$ blocks (see the next paragraph), these values became $179 \mathrm{~ms}$ and 0.06 , respectively.

Todd's (1981) original experiment is extremely timeconsuming. In order to simplify subsequent data collection, effects of the procedure were tested before substantial changes were introduced. For our second prestudy, only five different $\Delta t_{\mathrm{A}} \mathrm{s}(20 \sim 200 \mathrm{~ms})$ were used, assuming a difference threshold to lie somewhere within this range. Conditions $\Delta R=0$ and $\Delta v=0$ were eliminated, and blocks with different $\Delta t_{\mathrm{A}} \mathrm{s}$ of 50 trials each were ordered randomly. Thus, the total number of trials could be reduced to 250 . One new participant attained a JND of $174 \mathrm{~ms}$ (Weber fraction: 0.06). Two other participants, however, performed at chance level at all of the $\Delta t_{\mathrm{A}} \mathrm{s}$.

For our third prestudy, trials were drawn at random from the blocks defined in the second prestudy. Two of the observers who had served for Prestudies 1 and 2 both now attained a JND of $98 \mathrm{~ms}$ (Weber fraction: 0.03), again indicating a gain from perceptual learning. The other two participants from Prestudy 2, however, once again performed at chance level. One of them explained that her response strategy had been to always select the apparently larger object, thus ignoring velocity information. Obviously, such a strategy must lead to chance performance in a $\Delta t_{\mathrm{A}}$ task with $\Delta R \neq 0$.

Taken together, our prestudies suggested that fewer trials than were used by Todd (1981) suffice for determining $t_{\mathrm{A}}$ difference thresholds, if observers are sensitive to differences in optic flow velocities at all. Conversely, differences in projected size sometimes seem to have distracted from the task (cf. the "size arrival effect" described by DeLucia, 1991, 2004). For our main experiment, we therefore reduced the differences in size and velocity while at the same time making velocity information more salient and size information less prominent. 


\section{Main experiment}

On the basis of the theoretical considerations presented in the introduction, we constructed new stimuli (Fig. 1), and on the basis of the findings and observations from our prestudies, we developed a new experimental regime (Table 2). For our main experiment, in a $2 \times 2$ design, Todd's (1981) original stimuli and our new ones were factorially crossed with experimental regimes-namely, our new regime and the second, abbreviated version of Todd's regime as used in our third prestudy.

\section{Method}

Participants A group of 40 observers, ten for each cell of our design, participated. The participants were recruited on campus, and were - in balanced proportion-psychology or physics undergraduates. None had taken part in a $t_{\mathrm{A}}$ experiment before.

Stimuli As we already indicated in the introduction, two amorphous clouds, composed of 24 random dots each, were constructed. The clouds did not exhibit any obvious contours, and thus almost eliminated $\tau_{\mathrm{L}}{ }^{(2)}$ information while keeping the $\tau_{\mathrm{L}}{ }^{(1)}$ information from Todd's (1981) original dotted-outline squares, which were used as alternative stimuli. For reasons of comparability, as in the case of Todd's stimuli, the dots of our new stimuli did not change size during approach.

Experimental regimes In order to avoid the possible adaptation effects that had been reported by the observer of our complete replication of Todd's (1981) Experiment 1, in our modified experimental regime there was no fixed standard, and all variables except $\Delta t_{\mathrm{A}}$ were drawn at random from predefined ranges. To counter delusive effects of object size, a narrow range $(0.15 \sim 0.25 \mathrm{~m})$ was chosen for this variable. Velocities were sampled in pairs from ecologically meaningful ranges: $1.19 \sim 1.31 \mathrm{~m} \cdot \mathrm{s}^{-1}$ (pedestrian), $3.56 \sim 3.94 \mathrm{~m} \cdot \mathrm{s}^{-1}$ (bicycle), $10.69 \sim 11.81 \mathrm{~m} \cdot \mathrm{s}^{-1}$ (car in town), and 26.38 $29.16 \mathrm{~m} \cdot \mathrm{s}^{-1}$ (car on highway). $t_{\mathrm{A}}$ varied between $\sim 1.5$ and $\sim 2.5 \mathrm{~s}$, and $\Delta t_{\mathrm{A}}$ varied in steps of $25 \mathrm{~ms}$ between $\sim 50$ and $\sim 150 \mathrm{~ms}$. The procedure remained $2 \mathrm{AFC}$ for 250 trials, which were drawn according to the scheme used in Prestudy 3. The abbreviated version of Todd's regime that had been used in the latter prestudy was used for comparison.

\section{Results and discussion}

The mean difference thresholds-computed as half of the difference between the $25 \%$ and $75 \%$ points on fitted psychometric functions (cf., again, Wichmann \& Hill, 2001) - are listed according to conditions in Table 3. For our observers, Todd's (1981) abbreviated experimental regime proved much more difficult than our revision of it, $F(1$, $34)=27.118, p<.001, \eta^{2}=.444$. We found no main effect of stimulus variants, and no significant interaction between experimental regimes and stimuli (both $F_{\mathrm{S}}<1$ ).

For two of the participants, psychometric functions could not be fitted. Although this can partly be blamed on our choice of a narrow range of $\Delta t_{\mathrm{A}}$, it also reflects the original experimental regime's difficulty, because fits worked well for all observers who had been tested under the modified regime. As is evident from the standard deviations, there were tremendous interindividual differences: For our new experimental regime, the estimated thresholds ranged between 69.25 and $374.69 \mathrm{~ms}$, and for the abbreviated Todd regime, between 152.83 and $973.66 \mathrm{~ms}$.

Since 2 AFC decisions were not generally determined by projected object size or its rate of expansion at the time of the observers' responses, Todd (1981, p. 801: Table 1) reasoned that his participants probably used the ratio of these variables (i.e., $\tau$ ), which, as we explained in the introduction, specifies $t_{\mathrm{A}}$ (Lee, 1974; the differentiation between the three types of $\tau$ had not yet been published at the time Todd did his experiments; the $\tau$ implied was a special case of $\tau_{\mathrm{L}}{ }^{(1)}$, which referred to the side lengths of the squares used as stimuli; also, Todd's analysis was in terms of image sizes, not visual angles - which are equivalent in this case, however). We drew up the same statistics, and additionally counted the number of trials for which an object's larger visual angle $(\theta)$ or larger rate of angular change $(\mathrm{d} \theta / \mathrm{d} t)$ provided valid information about earlier arrival (Table 4). This also afforded a control of the randomization routine that we had used. It turned out that trials under Todd's (1981) abbreviated experimental regime were almost balanced with regard to whether the apparently larger or smaller object, or the apparently faster or slower one, would be the first to arrive, but that our new regime was biased in favor of the larger and faster (or closer) object. ${ }^{5}$ Despite this confound, it is obvious from Table 4 that observers relied on visual angle information most of the time-except for the cloud stimuli presented under our new experimental regime, $F(3,36)_{\theta}=24.785$, $p<.001, \eta^{2}=.674 ; F(3,36)_{\mathrm{d} \theta / \mathrm{d} t}=19.204, p<.001, \eta^{2}=.615$.

Unfortunately, the interpretation of the aforementioned statistics is not straightforward, because visual angles and their derivatives, under ecological conditions, are unavoidably correlated - which correlation also extends to $\tau$ (cf. Regan \& Hamstra, 1993; Schrater, Knill, \& Simoncelli, 2001, for ways to unconfound these variables). Therefore,

\footnotetext{
${ }^{5}$ Note that "apparently faster or slower" does not imply truly faster or slower, because angular velocities are affected by both objective velocity and distance.
} 
Table 3 Mean difference thresholds and standard deviations according to the stimuli and experimental regimes

\begin{tabular}{lll}
\hline Experimental Regimes & \multicolumn{2}{l}{ Stimuli } \\
\cline { 2 - 3 } & Dotted Squares & Dot Clouds \\
\hline Todd's abbreviated regime & $372.89 \mathrm{~ms}\left(n_{4}=9\right)$ & $426.32 \mathrm{~ms}\left(n_{3}=9\right)$ \\
& $S D: 169.15 \mathrm{~ms}$ & $S D: 246.37 \mathrm{~ms}$ \\
Our regime & $136.00 \mathrm{~ms}\left(n_{2}=10\right)$ & $138.24 \mathrm{~ms}\left(n_{1}=10\right)$ \\
& $S D: 87.63 \mathrm{~ms}$ & $S D: 62.13 \mathrm{~ms}$ \\
\hline
\end{tabular}

The indices of $n$ denote the four independent subsamples

Todd's (1981) conclusion, that his observers were sensitive to $\tau$, seems premature. For our experiment, the percentages of trials in which participants' responses were consistent with the use of $\theta$ or $\mathrm{d} \theta / \mathrm{d} t$ were always greater than the percentage referring to $\tau$ (Table 4). On the other hand, the percentages were not too different for the cloud stimuli under our new experimental regime, and also, the percentages of trials for which responses were consistent with the use of $\tau$ were generally greater for the new regime than they had been in Todd (1981), $F(3,36)_{\tau}=16.865, p<.001, \eta^{2}=.584$. Taken together, these findings suggest that by reducing the size difference between objects $(\Delta R)$, we were successful at emphasizing velocity and optic flow information, so that under our experimental regime - with the cloud stimuli, in particular-observers may have used $\tau_{\mathrm{L}}{ }^{(1)}$-related information more readily than in the other conditions.

\section{General discussion}

Here we have shown that $t_{\mathrm{A}}$ difference thresholds are strongly affected by experimental regimes. This effect seems closely related to DeLucia's (1991) size arrival effect, because changing the relative sizes of objects was one of our major modifications of Todd's (1981) original regime. The absence of a main effect of stimulus variants, on the other hand, may indicate great flexibility of mechanisms in the extraction of information from optic flow. Yet, we also have to consider the possibility that many observers on many trials may not have used $\tau$-type variables at all.

Several authors (e.g., DeLucia, 2004) have suggested that observers in a $t_{\mathrm{A}}$ situation may use multiple sources of information (see Yan, Lorv, Li, \& Sun, 2011, for a recent review and perspective). One of the virtues of Todd's (1981) stimulus scenario is that it isolates - to a fair approximation - those variables that theoretically suffice to deal successfully with impending, head-centered collisions: one or more visual angles $\left(\theta_{i}\right)$, those angles' rates of change $\left(\mathrm{d} \theta_{i} / \mathrm{d} t\right)$, and the ratios of these terms $\left(\tau_{i}\right.$; cf. Regan \& Hamstra, 1993, who presented evidence that well-practiced observers may be able to utilize any of these sources of information independently from the others; Schiff, Caviness, \& Gibson, 1962; and Wang \& Frost, 1992, who used even simpler, single-object scenarios, apt for animal research). Crucially, in all of these simulations, there was no information about distance. This entails that a "cognitive" strategy to compute $t_{\mathrm{A}}$ by dividing perceived distance and perceived velocity is unlikely to be successful-if it is applied at all (Tresilian, 1991). Still, observers may attempt to infer distances from apparent object sizes (Gibson, 1950) and respond to distance to arrival $\left(d_{\mathrm{A}}\right)$ instead of $t_{\mathrm{A}}$ (see Liu, Niu, \& Wang, 2008, for evidence of accordingly tuned neurons in the pigeon, although $d_{\mathrm{A}}$ is believed to be obtained from velocity- and $\tau_{\mathrm{G}}$-sensitive neurons, on the basis of the equality of $d=v \cdot t$ ). In a $2 \mathrm{AFC}$ scenario, such a response strategy would show up as overreliance on the difference of visual angles $\left(\Delta \theta_{i, j}\right)$ - exactly what we found.

Eventually, how do we account for the individual differences between our observers? Under our simplified experimental

Table 4 Proportions of trials for which, at the time of participants' responses, the larger visual angle of one object, or the larger angular velocity of one object, provided valid information for correct responses,

and proportions of trials on which participants selected this object to be the first to arrive, split up into actually correct versus erroneous decisions

\begin{tabular}{|c|c|c|c|c|c|c|c|c|c|c|}
\hline \multirow[t]{2}{*}{ Regime and Stimulus } & \multirow{2}{*}{$\begin{array}{l}\text { Larger } \theta= \\
\text { Valid Cue }\end{array}$} & \multirow{2}{*}{$\begin{array}{l}\text { Larger } \theta \\
\text { Selected }\end{array}$} & \multicolumn{2}{|l|}{ Answer } & \multirow{2}{*}{$\begin{array}{l}\text { Larger } \mathrm{d} \theta / \mathrm{d} t= \\
\text { Valid Cue }\end{array}$} & \multirow{2}{*}{$\begin{array}{l}\text { Larger } \mathrm{d} \theta / \mathrm{d} t \\
\text { Selected }\end{array}$} & \multicolumn{2}{|l|}{ Answer } & \multirow{2}{*}{$\begin{array}{l}\text { Smaller } \tau \\
\text { Selected }\end{array}$} & \multirow{2}{*}{$\begin{array}{l}\text { Correlations } \\
\text { Between } \theta \\
\text { and } \mathrm{d} \theta / \mathrm{d} t\end{array}$} \\
\hline & & & Correct & Incorrect & & & Correct & Incorrect & & \\
\hline \multicolumn{11}{|l|}{ Todd's Regime } \\
\hline Squares & .59 & .94 & .55 & .39 & .64 & .90 & .56 & .35 & .57 & .65 \\
\hline Clouds & .59 & .94 & .55 & .39 & .63 & .92 & .56 & .36 & .57 & .61 \\
\hline \multicolumn{11}{|l|}{ Our New Regime } \\
\hline Squares & .74 & .91 & .68 & .23 & .83 & .86 & .70 & .16 & .71 & .33 \\
\hline Clouds & .80 & .77 & .64 & .14 & .89 & .76 & .67 & .09 & .70 & .32 \\
\hline
\end{tabular}

The last column lists correlations between $\theta$ and $\mathrm{d} \theta / \mathrm{d} t \cdot \theta=$ plane visual angle of objects, referred to maximum horizontal diameter. $\mathrm{d} \theta / \mathrm{d} t=$ instantaneous change of $\theta$ at time of participants' responses. $\tau=\theta \div \mathrm{d} \theta / \mathrm{d} t . \tau$ was always a valid cue, and if the corresponding answers were selected, they were necessarily correct. The proportions "selected" do not add up across $\theta$ and $\mathrm{d} \theta / \mathrm{d} t$ because the variables were correlated. The proportions "correct" and "incorrect" add up to the proportions "selected" 
regime, several participants attained $t_{\mathrm{A}}$ difference thresholds almost as low as those that were achieved by Todd's (1981) trained observers. Others, however, especially when tested under variants of Todd's original regime, failed completely. As has been confirmed by other researchers (personal communications by F. T. J. M. Zaal, July 2, 2007, and E. Brenner, May 11, 2010), self-reported $t_{\mathrm{A}}$-critical activities like sports, driving, or computer game proficiency do not in general predict these individual differences. Whether they are related to deficits in basic skills of discriminating visual angles and their changes is an issue to be investigated in future studies.

In sum, it seems that the discrepancies between Todd's (1981) original data concerning $\Delta t_{\mathrm{A}}$ thresholds and later findings are best explained by effects of the experimental regimes, and that these effects in turn come about because observers tend to base decisions about time to arrival more on apparent object sizes than on temporal cues.

Author note R.B. is now at Université Paul Sabatier, Toulouse, France. The work presented in this article was supported by a grant of the Deutsche Forschungsgemeinschaft to H.H. (HE 2122/6-1: "Kontaktzeitschätzung im Kontext"). R.B. was supported by the Alexander-von-HumboldtStiftung. We thank Agnes Münch for programming experiments, and Elsa $\mathrm{Krau} ß$ and Bernhard Both for support in analyzing the data. Parts of this work were presented at ECVP 2009 and VSS 2010. Correspondence concerning this article should be addressed to Klaus Landwehr, Psychologisches Institut, Universität Mainz, 55099 Mainz, Germany. E-mail: landweh@uni-mainz.de

\section{References}

Beverley, K. I., \& Regan, D. (1979). Visual perception of changing size: The effect of object size. Vision Research, 19, 1093-1104.

Beverley, K. I., \& Regan, D. (1980). Visual sensitivity to the shape and size of a moving object: Implications for models of object perception. Perception, 9, 151-160.

Bootsma, R. J., \& Craig, C. (2002). Global and local contributions to the optical specification of time to contact: Observer sensitivity to composite tau. Perception, 31, 901-924.

Bootsma, R. J., \& Oudejans, R. R. D. (1993). Visual information about time-to-collision between two objects. Journal of Experimental Psychology: Human Perception and Performance, 19, 1041-1052.

Bootsma, R. J., \& van Wieringen, P. C. W. (1990). Timing an attacking forehand drive in table tennis. Journal of Experimental Psychology: Human Perception and Performance, 16, 21-29.

DeLucia, P. R. (1991). Pictorial and motion-based information for depth perception. Journal of Experimental Psychology: Human Perception and Performance, 17, 738-748.

DeLucia, P. R. (2004). Multiple sources of information influence time-tocontact judgments: Do heuristics accommodate limits in sensory and cognitive processes? In H. Hecht \& G. J. P. Savelsbergh (Eds.), Timeto-contact (pp. 243-285). Amsterdam, The Netherlands: Elsevier.

DeLucia, P. R., Kaiser, M. K., Bush, J. M., Meyer, L. E., \& Sweet, B. T. (2003). Information integration in judgements of time to contact. Quarterly Journal of Experimental Psychology, 56A, 1165-1189.

Fechner, G. T. (1860). Elemente der Psychophysik [Elements of psychophysics, 2 vols.]. Leipzig, Germany: Breitkopf \& Härtel.
Giachritsis, C. D., \& Harris, M. G. (2005). Global versus local image expansion in estimating time-to-contact from complex optic flow. Perception, 34, 577-585.

Gibson, J. J. (1950). The perception of the visual world. Boston, MA: Houghton Mifflin.

Harris, M. G., \& Giachritsis, C. D. (2000). Coarse-grained information dominates fine-grained information in judgments of time-tocontact from retinal flow. Vision Research, 40, 601-611.

Hosking, S. G., \& Crassini, B. (2011). The influence of optic expansion rates when judging the relative time to contact of familiar objects. Journal of Vision, 11(6), 20. doi:10.1167/11.6.20

Jacobs, D. M., \& Díaz, A. (2010). Judgements of time to contact are affected by rate of appearance of visible texture. Quarterly Journal of Experimental Psychology, 63, 1041-1048.

Jones, F. N. (1971). History of psychophysics and judgment. In E. C. Carterette \& M. P. Freidman (Eds.), Handbook of perception (Vol. 2, pp. 1-22). New York, NY: Academic Press.

Kaiser, M. K., \& Mowafy, L. (1993). Optical specification of time-topassage: Observers' sensitivity to global tau. Journal of Experimental Psychology: Human Perception and Performance, 19, $1028-1040$.

Kanizsa, G. (1979). Organization in vision. New York, NY: Praeger.

Kebeck, G., \& Landwehr, K. (1992). Optical magnification as event information. Psychological Research, 54, 146-159.

Kim, N.-G., \& Grocki, M. J. (2006). Multiple sources of information and time-to-contact judgments. Vision Research, 46, 1946-1958.

Landwehr, K. (2004). Textured tau. In H. Hecht \& G. J. P. Savelsbergh (Eds.), Time-to-contact (pp. 229-242). Amsterdam, The Netherlands: Elsevier.

Lee, D. N. (1974). Visual information during locomotion. In R. B. McLeod \& H. L. Pick (Eds.), Perception: Essays in honor of James J. Gibson (pp. 250-267). Ithaca, NY: Cornell University Press.

Lee, D. N. (1976). A theory of visual control of braking based on information about time-to-collision. Perception, 5, 437-459.

Lee, D. N., \& Young, D. S. (1985). Visual timing of interceptive action. In D. J. Ingle, M. Jeannerod, \& D. N. Lee (Eds.), Brain mechanisms and spatial vision (pp. 1-30). Dordrecht, The Netherlands: Nijhoff.

Liu, R.-F., Niu, Y.-Q., \& Wang, S.-R. (2008). Thalamic neurons in the pigeon compute distance-to-collision of an approaching surface. Brain, Behavior and Evolution, 72, 37-47.

López-Moliner, J., Brenner, E., \& Smeets, J. B. J. (2007). Effects of texture and shape on perceived time to passage: Knowing "what" influences judging "when. Perception \& Psychophysics, 69, 887-894.

Macmillan, N. A., \& Creelman, C. D. (2005). Detection theory: A user's guide (2nd ed.). Mahwah, NJ: Erlbaum.

McKee, S. P. (1981). A local mechanism for differential velocity detection. Vision Research, 21, 491-500.

Morgan, M. J., Watamaniuk, S. N. J., \& McKee, S. P. (2000). The use of an implicit standard for measuring discrimination thresholds. Vision Research, 40, 2341-2349. doi:10.1016/S0042-6989(00)00093-6

Norman, J. F., Pattison, K. F., Norman, H. F., Craft, A. E., Wiesemann, E. Y., \& Taylor, M. J. (2008). The role of explicit and implicit standards in visual speed discrimination. Perception, 37, 889-901.

Oberfeld, D., Hecht, H., \& Landwehr, K. (2011). Effects of task-irrelevant texture motion on time-to-contact judgments. Attention, Perception, \& Psychophysics, 73, 581-596. doi:10.3758/s13414-010-0040-3

Pentland, A. (1980). Maximum likelihood estimation: The best PEST. Perception \& Psychophysics, 28, 377-379.

Regan, D. (2012). Vision and cricket. Ophthalmic and Physiological Optics, 32, 257-270.

Regan, D., \& Hamstra, S. J. (1993). Dissociation of discrimination thresholds for time to contact and rate of angular expansion. Vision Research, 33, 447-462.

Roelfsema, P. R. (2006). Cortical algorithms for perceptual grouping. Annual Review of Neuroscience, 29, 203-227. doi:10.1146/ annurev.neuro.29.051605.112939 
Sasaki, Y. (2007). Processing local signals into global patterns. Current Opinion in Neurobiology, 17, 132-139.

Schiff, W., Caviness, J. A., \& Gibson, J. J. (1962). Persistent fear responses in rhesus monkeys to the optical stimulus of "looming. Science, 136, 982-983.

Schiff, W., \& Oldak, R. (1990). Accuracy of judging time to arrival: Effects of modality, trajectory, and gender. Journal of Experimental Psychology: Human Perception and Performance, 16, 303316.

Schrater, P. R., Knill, D. C., \& Simoncelli, E. P. (2001). Perceiving visual expansion without optic flow. Nature, 410, 816-819.

Simpson, W. A. (1988). Depth discrimination from optic flow. Perception, 17, 497-512.

Todd, J. T. (1981). Visual information about moving objects. Journal of Experimental Psychology: Human Perception and Performance, 7, 795-810.
Tresilian, J. R. (1991). Empirical and theoretical issues in the perception of time to contact. Journal of Experimental Psychology: Human Perception and Performance, 17, 865-876.

Wang, Y.-C., \& Frost, B. J. (1992). Time to collision is signalled by neurons in the nucleus rotundus of pigeons. Nature, 356, 236-238.

Warren, W. H. (1998). The state of flow. In T. Watanabe (Ed.), High-level motion processing. Computational, neurobiological, and psychophysical perspectives (pp. 315-358). Cambridge, MA: MIT Press.

Westheimer, G., \& Li, W. (1996). Classifying illusory contours by means of orientation discrimination. Journal of Neurophysiology, 75, 523-528.

Wichmann, F. A., \& Hill, N. J. (2001). The psychometric function: II. Bootstrap-based confidence intervals and sampling. Perception \& Psychophysics, 63, 1314-1329. doi:10.3758/BF03194545

Yan, J.-J., Lorv, B., Li, H., \& Sun, H.-J. (2011). Visual processing of the impending collision of a looming object: Time to collision revisited. Journal of Vision, 11(12), 7. doi:10.1167/11.12.7 